\title{
Why Have Immunization Efforts in Pakistan Failed to Achieve Global Standards of Vaccination Uptake and Infectious Disease Control?
}

This article was published in the following Dove Press journal: Risk Management and Healthcare Policy

\author{
Mahreen Butt $\mathbb{D}^{\prime}$ \\ Raihan Mohammed (D) ${ }^{2}$ \\ Eman Butt $\mathbb{D}^{2}$ \\ Sundas Butt $\mathbb{D D}^{3}$ \\ Jinpo Xiang ${ }^{4}$
}

'Institute for Global Health, University College London, London, UK;

${ }^{2}$ Department of Medicine, University of Cambridge, Cambridge, UK; ${ }^{3}$ Faculty of Medicine, University of Liverpool, Liverpool, UK; ${ }^{4}$ Faculty of Medicine, Imperial College London, London, UK
Correspondence: Raihan Mohammed Department of Medicine, University of Cambridge, Hills Road, Cambridge CB2 OSP, UK

Email rm758@cam.ac.uk

\begin{abstract}
Immunization is one of the most successful public health initiatives in recent times. It is, therefore, worrying to learn the level of under-vaccination in Pakistan. Diseases that have been successfully eliminated through the aid of vaccination in other countries have not been eliminated in Pakistan. The reasons for this vary and show the uniqueness of the economic, healthcare and environmental landscape of Pakistan, through which public health programmes need to be implemented. The "Expanded Programme of Immunization" (EPI) is the main programme through which routine immunization is provided to the public. Within Pakistan, it has encountered many problems since its inception. This includes logistical obstacles, inefficient health worker attitudes, parental and female awareness, and education, the influence of religious community leaders and the complications that accompany conflict. When compared to globally standardised targets for immunization, Pakistan is trailing behind. Not achieving these targets is worrying from both a global perspective and within the national healthcare landscape of Pakistan. Research is necessary to bring together findings on the failings of routine immunization and polio campaigns; there are many intersecting factors that global health bodies and the Department of Health in Pakistan must address in order to relieve the burden of vaccine-preventable diseases (VPDs).
\end{abstract}

Keywords: Pakistan, vaccination, immunization, coverage, Polio, EPI, WHO

\section{Introduction}

Here, we examine the ways in which various immunization efforts, for a range of vaccine-preventable diseases, have achieved success or failure in Pakistan and the reasons why this plays a part in the VPD burden of Pakistan. Mangrio emphasises how "immunization is the most cost-effective public health intervention that has had the greatest impact on health of the people". 1 Therefore, the importance of countries to successfully implement immunization programs is imperative for the health of the public; making Immunization an important facet of public health. Due to routine immunization worldwide, millions of children are saved from illness and death by vaccine-preventable diseases. Yet in spite of this successful public health initiative, Pakistan has not yet assimilated this enterprise into their own health profile with the same level of proficiency. Vaccine-preventable diseases are a major cause of death amongst children, especially within developing countries, accounting for more than one million deaths annually. ${ }^{2}$ For example, 90,000 children still suffer from paralytic polio and measles accounts for 20,000 child deaths annually. ${ }^{3}$ This high mortality from VPDs is despite the fact that vaccinations are generally 
inexpensive, easy to administer and a priority of healthcare systems. ${ }^{1}$ We aim to analyze the obstacles that Pakistan presents for the failures to adequately immunize its population.

The failures to successfully immunize the population of Pakistan has impacted child mortality in the country and is an important area of research for the progression of child healthcare. Children who are under the age of five make up $15 \%$ of the population of Pakistan. Unfortunately, this demographic makes up $50 \%$ of the mortality rate in this country. For comparison, the world average for underfive mortality as a percentage of overall mortality is around $8 \%{ }^{4}$ Following on from this, the under-five mortality rate in Pakistan is $81 \%$ according to the World Bank's most recent data as of $2015 .^{5}$ In order to achieve Millennium Development Goal Four, Pakistan must reduce this under-five mortality rate by nearly half to $45 \%{ }^{6}$ The need for interventions that specifically target child health such as vaccination is, therefore, important for Pakistan if they are to reduce mortality and improve the health status of the nation. A significant $70 \%$ of childhood death is due to infectious disease. ${ }^{7}$ In some areas of Pakistan, the introduction of immunization programmes increased coverage from $5 \%$ to $84 \%$. Despite this, $58 \%$ of children at risk of disease are currently unimmunized. ${ }^{4}$ Pakistan has the third highest burden of child mortality and is not achieving Millennium Development Goal Four at an acceptable rate, meaning it is trailing behind for child mortality. For this reason, there has been an increased focus on how best to tackle this. A multi-faceted approach must be met, in which social determinants of health such as female literacy and poverty are addressed, with a simultaneous scaling up of evidence-based interventions such as vaccinations. ${ }^{8}$

The extreme health burden of VPDs in Pakistan has wider, secondary impacts on the development of the nation. In general, infectious diseases reflect a huge economic burden on developing countries. For hospitalised cases of pneumonia - an episode can cost up to 235 dollars and a case of meningitis can cost over 2000 dollars in Pakistan. ${ }^{9}$ These are economic burdens that fall under the individual in the majority of cases as out-of-pocket payment is overwhelmingly the main method of health financing.

Pakistan's Expanded Programme of Immunization (EPI) was established in order to tackle the main vaccinepreventable diseases in Pakistan. These include poliomyelitis, maternal and neonatal tetanus and measles. ${ }^{10}$ The EPI in Pakistan is supported by the "Global Alliance for Vaccines and Immunization" (GAVI), which is a publicprivate global health partnership with the aim to increase immunization coverage in low- and middle-income countries. ${ }^{11}$ Through this, we can see that multiple players are involved in routine immunization and polio campaigns, making the elimination of VPDs a priority for Pakistan and the international global health community. GAVI primarily acts via Civil Society Organisations (CSOs) at the district level in Pakistan. This interplay between both the international bodies, the government of Pakistan and the communities, through which they have to operate, needs to also be analyzed as it brings up obstacles to adequate service delivery and uptake of vaccines. Variables that are connected to vaccine coverage include female literacy, household income, supply of vaccines, number of vaccinators and monitoring of this health initiative. $^{12}$ On a further level - nurse and lady's health worker (LHW) density was positively associated with vaccination coverage whereas doctor density did not have such a strong correlation. ${ }^{2}$ Data differ on whether household income is really a contributing factor to vaccine uptake however female literacy has shown to have a significant effect. Furthermore, in Pakistan estimates of children vaccinated between one and two years varies from $59 \%$ to $73 \%$ on all routine immunizations, ${ }^{13}$ and the uptake of vaccines varies throughout the country.

The purpose of this literature review is to assess why Pakistan has not succeeded with regards to immunization, comparable to standards set by global health authorities. An analysis into the current state of vaccination across Pakistan is needed as well as an analysis into why these rates do not suffice global standards of public health. Ultimately, we hope to provide recommendations as to how best to overcome why immunization efforts in Pakistan failed to achieve global standards of public health.

\section{The Expanded Programme of Immunization}

EPI was set up by the World Health Organisation (WHO) as a global initiative, in many developing countries, in order to address VPDs. This programme is implemented globally, and different national logistics affect how it is applied. The WHO acknowledges this and implements the EPI to be as countryspecific as possible. However, although similar methods and facilities are put into place within each country "local 
realities and national policies" must be addressed for the success of individual immunization programmes. ${ }^{14}$ In 2012, 194 member states of the World Health Assembly endorsed the "Global Vaccine Action Plan" (GVAP), which laid out instructions that are both for the global and country-specific perspective. It is significant to note that Pakistan is detailed as a "priority country" with regard to their immunization. ${ }^{15}$ According to the Official Secretariat Report of the GVAP; global targets that Pakistan is trying to achieve include:

(a) "Achieve 90\% national coverage and $80 \%$ in every district with three doses of diphtheria-tetanus-pertussis containing vaccines".

(B) "Achieve 90\% national coverage and $80 \%$ in every district with all vaccines included in the national schedule."

(C) achieve a world free of poliomyelitis. ${ }^{15}$

In order to achieve these GVAP targets by 2020, "the current pace of global progress must change". 15 The importance of EPI in Pakistan cannot be underestimated. It is the main source of vaccination for the general public. Understanding its concept and implementation in Pakistan is necessary to realising its failings and hence areas of improvement.

The success and failures of the EPI in Pakistan have been shaped by the social, political and environmental landscape. The main aim of the programme when it was set up in 1978 was to prevent deaths, and after initial success, set to eliminate polio by $2000^{16,17}$ and measles and neonatal tetanus by 2015. ${ }^{18}$ The EPI, as mentioned, is the main provider of vaccinations in Pakistan and can be defined as a publicprivate partnership, in which pharmaceutical companies, UNICEF, the WHO and national governments work together to implement the immunization programme. ${ }^{19}$ It is the main programme that provides vaccination coverage within Pakistan, where the cost of immunising a child fully is around 15 US dollars. ${ }^{20}$ Currently, EPI reaches 5.8 million children under the age of one. It is important to remember that this means these 5.8 million children have been vaccinated against the "standard" nine vaccine-preventable diseases. In addition to this, there are 5.9 million women who are pregnant that have been vaccinated against tetanus, which is done in order to protect both them and their newborns against tetanus. However, 30 million children are targeted with polio vaccination, due to the international focus and goal to eliminate polio worldwide. ${ }^{21}$ See Table 1.

There are over 6000 "fixed" centres of immunization currently running within Pakistan and an extra one million mobile outreach services providing similar facilities for harder to reach areas. ${ }^{22}$ There are over 10,000 vaccine personnel including Lady Health Workers and paramedics who provide vaccines to patients. However, as a system,

Table I Pakistan's EPI Vaccination Schedule. This Programme of Immunization Is the Main Programme That Provides Vaccination Coverage Within Pakistan. Currently, EPI Reaches 5.8 Million Children Under the Age of One. To Be Fully Vaccinated Against the Nine VPDs, a Child Needs Only Six Visits Over the Course of Two Years

\begin{tabular}{|c|c|c|c|c|}
\hline Disease & Causative Agent & Vaccine & Doses & Age of Administration \\
\hline Childhood TB & Bacteria & BCG & 1 & Soon after birth \\
\hline Poliomyelitis & Virus & $\begin{array}{l}\text { OPV } \\
\text { IPV }\end{array}$ & $\begin{array}{l}4 \\
\text { I }\end{array}$ & $\begin{array}{l}\text { OPV0: soon after birth } \\
\text { OPVI: } 6 \text { weeks } \\
\text { OPV2: } 10 \text { weeks } \\
\text { OPV3: } 14 \text { weeks } \\
\text { IPV-I: I4 weeks }\end{array}$ \\
\hline Diphtheria & Bacteria & \multirow{5}{*}{$\begin{array}{l}\text { Pentavalent vaccine } \\
(\mathrm{DTP}+\mathrm{Hep} \mathrm{B}+\mathrm{Hib})\end{array}$} & \multirow[t]{5}{*}{3} & \multirow{5}{*}{$\begin{array}{l}\text { Penta I: } 6 \text { weeks } \\
\text { Penta2: } 10 \text { weeks } \\
\text { Penta3: } 14 \text { weeks }\end{array}$} \\
\hline Tetanus & Bacteria & & & \\
\hline Pertussis & Bacteria & & & \\
\hline Hepatitis B & Virus & & & \\
\hline Hib pneumonia and meningitis & Bacteria & & & \\
\hline Measles & Virus & Measles & 2 & $\begin{array}{l}\text { Measles 1: } 9 \text { months } \\
\text { Measles2: I5months }\end{array}$ \\
\hline Diarrhoea due to rotavirus & Virus & *Rotavirus & 2 & $\begin{array}{l}\text { Rota I: } 6 \text { weeks } \\
\text { Rota 2: } 10 \text { weeks }\end{array}$ \\
\hline
\end{tabular}


the local vaccinators are not directly supervised by national EPI managers. The supply chain begins with EPI managers who supply EPI products to the provinces. The vaccinator team, comprised of a driver and dedicated vaccine logistics specialists, is then responsible for distributing products to the lower levels of the supply chain. Instead of direct guidance by EPI managers, vaccinators are required to pick up vaccines and other supply chain tasks as part of their overall immunization responsibilities, and are directly responsible to Director-General of Health workers, a separate line director. In order to create an efficient system for vaccine distribution, the EPI representatives need to understand the realities in local districts, which is prevented by this hierarchy. ${ }^{23}$

The main three goals of the EPI in the contemporary context are the elimination of polio, measles and neonatal tetanus. ${ }^{10}$ Although these figures may seem impressive, there are other convening factors that relate to actual vaccination uptake. The progress that the EPI has made has been assessed by independent parties - namely the WHO and UNICEF. These organisations reported an increase in vaccination coverage but at a "slow rate". 24 Ultimately, however, this was not enough to ensure elimination or in other words to reach any targets set in place.

\section{Current Status of Vaccination in Pakistan}

The overall immunization coverage is an important measurement for international bodies to assess the level of success of vaccination programmes in Pakistan. It is a standard that can easily define success or failure against targets, although overall it may be a measurement that is too broad to reveal the reasons why immunization efforts have not succeeded. Immunization coverage in Pakistan has seen a unique trajectory in the past few decades.

According to estimates, EPI coverage is $80 \%$ for Bacillus Calmette-Guérin (BCG), 65\% for combined diphtheria, pertussis, and tetanus (DPT3) and polio and $67 \%$ for measles. ${ }^{25}$ Tetanus is the second leading cause of death of infants in Pakistan (21.6\% of all deaths). ${ }^{25}$ For individual vaccines, different research reports different levels of immunization. One such study assessed these differences and reported that "The coverage of individual vaccines was $76 \%$ for BCG, $61 \%$ for DPT 1, 49\% for DPT 2 , $45 \%$ for DPT 3 and about $27 \%$ for measles". ${ }^{25,26}$ However, according to UNICEF in its report "State of the World Children 2004", in comparison, BCG coverage is less at
$67 \%$, DPT 3 is much higher at $63 \%$ and measles also higher at $57 \%{ }^{27}$ A further study in Hyderabad, Pakistan, showed that in one to three-year-old infants, BCG coverage was reported as $72 \%$, DPT $1-3$ as $65 \%$ and measles as $41 \%{ }^{25,28}$ From these discrepancies, it is evident there is a problem with the reporting of vaccination status, which brings up problems when assessing the true level of success of Pakistan in this public health initiative. Also, even if the first doses of vaccines are completed - the subsequent necessary doses have a lower uptake which effectively renders vaccine schemes incompetent in their targets. Between 2000 and 2004 just over $10 \%$ of children who received the first dose of DPT went on to complete the full programme of vaccination up to the third dose (DPT3). ${ }^{29}$ Even basic data on overall coverage reveal a lot of where the failings of the immunization initiative lie. Data on this are very important. At the district level also, we can look at this to further analyze such disparities. See Table 2.

In 2011, the healthcare system of Pakistan shifted there was a devolution of health services from the federal to the provincial level. This means that since there has been a decentralisation of primary healthcare services, including and especially for vaccinations. This change caused "initial confusion around the roles and responsibilities of EPI staff and financing at all levels". ${ }^{15}$ Further to this, provincial governments have less resources and means to roll out intensive routine immunization campaigns as compared to nationwide initiatives.

Overall, immunization rates vary between provinces and they do not each follow the same trend for increasing or decreasing immunization coverage. Punjab, which is the biggest province within Pakistan, by earliest estimates had overall immunization coverage of $49 \%$. This gradually increased at every survey into $66 \%$ in 2003 to $84 \%$ in 2005 . However, by 2007 rates had dropped to $53 \%$, which is significant and

Table 2 EPI Coverage of Vaccine-Preventable Diseases Can Be Highly Varied Depending on the Area Within Pakistan and the Study Conducted. This Discrepancy and Lack of Confirmation with Regard to Vaccination Rates, Causes Difficulty in Assessing the Success of EPI

\begin{tabular}{|l|l|l|l|l|}
\hline Vaccine & $\begin{array}{l}\text { EPI } \\
\text { Coverage \% } \\
\text { (Siddiqui } \\
\text { et al 2006) }\end{array}$ & $\begin{array}{l}\text { EPI } \\
\text { Coverage \% } \\
\text { (Rehman } \\
\text { et al 20I7) }\end{array}$ & $\begin{array}{l}\text { EPI } \\
\text { Coverage } \\
\% \text { (Unicef } \\
\text { 2007) }\end{array}$ & $\begin{array}{l}\text { EPI } \\
\text { Coverage } \\
\% \text { (Zaidi } \\
\text { et al 2009) }\end{array}$ \\
\hline BCG & 80 & 76 & 67 & 72 \\
DPT3 & 65 & 45 & 63 & 65 \\
Measles & 67 & 27 & 57 & 41 \\
\hline
\end{tabular}


the reasons as to why must be assessed. Sindh saw a similar increase and subsequent decrease. In contrast to this, Balochistan, in the north of Pakistan, started with the highest immunization coverage at $60 \%$ in 1995 . Since then coverage fell until it was at $35 \%$ by 2007 , which is the lowest coverage for any province in Pakistan. ${ }^{24}$ A study carried out in Khyber Pakhtunkhwa (KPK) revealed a vaccination rate of $37.6 \%$ and $65 \%$ in urban and rural settings, respectively. A study conducted in nine Union Councils of sub-district Gambat, district Khairpur, Sindh showed a complete vaccination coverage of $71.7 \%$. Another study carried out in peri-urban Karachi revealed a vaccination rate of $44.8 \%$. Surveys conducted in Faisalabad and Nurpur Shahan regions of Punjab showed vaccination coverage of $63 \%$ and $77.4 \%$, respectively. ${ }^{30}$ District variation is an interesting phenomenon in Pakistan and owes to the fact that there is district levelbased healthcare, and there are differences in the culture and environment across the country. See Figure 1.

There are different ways in which immunization coverage has been assessed. Vaccination coverage is usually assessed via surveys which have been taken by different groups over the past years. The EPI collected data via surveys in the years 2001, 2003, and 2006. Other surveys such as the "Pakistan Integrated Household Survey" were taken in 1995, 1996, 1998, 1999, 2001, 2002. All surveys used the same definition of the fully vaccinated child which included; one dose of BCG, three oral polio vaccine (OPV) doses, up to one of the DPT3 vaccine and the one measles vaccine. This may contribute to the fact that overlapping surveys did provide similar results, which supports the accuracy of the data. However, one survey taken in 2004-2005, "The Pakistan Social and Living Standards Measurement Survey" reported much higher immunization coverage - of about $20-30 \%$ more than other surveys. ${ }^{24}$ The reasons for this are unknown. However, the disparities in data do bring to light problems with data collection and accuracy of numbers. It is worrying to learn that a majority of experts agree that many data collection on polio in Pakistan is counterfeit. $^{22}$ The reasons for this may be to meet daily targets in order to get more resources. However, it leads to the under-reporting of how many children have not been vaccinated, how many have acute flaccid paralysis and how many families refuse vaccination. ${ }^{22}$ This information is vital in order to understand the extent of under-vaccination.

However, with the advancements in digital health, this has shown to be promising for improving the monitoring of immunization activities. The advent of the Zindagi Mehfooz (Safe Life; ZM) Digital Immunization Registry in Pakistan, which is an Android phone-based system, allows vaccinators to digitally enrol and track the immunization status of their district. This also creates real-time access to data and easily generates reports. ${ }^{31}$ Automated

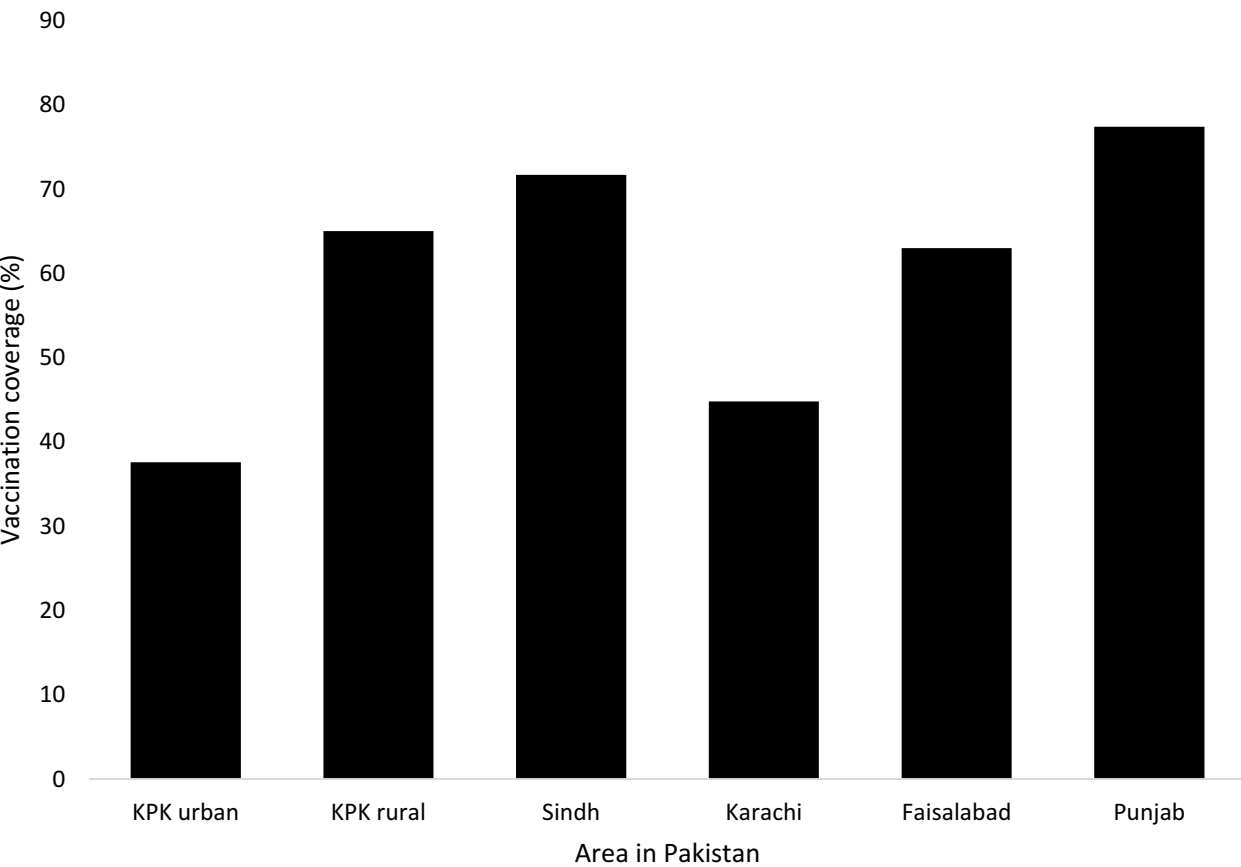

Figure I There is variation in vaccination coverage depending on the area within Pakistan; this is not only dependent on the province, but on the rural/urban status of the relevant area. 
SMS-based systems sent to the target population, which ask questions, such as "Did the vaccinator visit your house?" can easily track vaccine uptake. ${ }^{32}$ However, further research is needed to determine if these digital data are sufficiently representative of the true values of vaccine coverage. This relatively simple digital intervention is cheap, portable and easily accessible in Pakistan, due to the large population in developing countries that have access to mobile phones. This offers a huge potential for public health engagement and improves vaccination uptake and data collection.

Higher rates of vaccination were found in urban areas rather than in rural areas. ${ }^{33}$ This is important as more than $70 \%$ of Pakistan's 164 million population live in rural areas, ${ }^{6}$ and so a large fraction of the population of Pakistan are not benefiting from vaccinations. The disparities between these two demographics are significant even within the same province. For this reason, implementing a nationwide immunization programme has been met with less than great success, as the social, religious and economic narratives play out differently in these close-by yet different demographics.

The investigation into tetanus toxoid (TT) vaccine coverage showcases these differences well and has shown great disparities between rural and urban areas. Lambo insists that "a disparity exists in TT vaccination coverage and antenatal care between urban and rural areas due to access and utilization of health care services." This has led to great differences in TT coverage ranging from $60 \%$ to $74 \%$ across the country. ${ }^{21}$ Even though there may seem like there are many immunization centres throughout Pakistan, parents still report distance to immunization centres as one of the leading reasons as to why they do not take their children to get vaccinated. ${ }^{34}$ This may be because the 6000 immunization centres are not adequately distributed throughout the country. This disparity can be seen in Punjab; this area is known as the province with the most developed infrastructure, yet in $10 \%$ of areas, there is no fixed EPI centre. The number of vaccinators in areas is also not adequate.

Research shows that there should be two vaccinators per Union Council (UC). The real figure is there are 1.3 vaccinators per UC in all provinces except Sindh. The distance of vaccination centres may not seem like a vital issue; however, it affects whether people take their children to get vaccination to a great degree. If the vaccination centre was within a $12-\mathrm{km}$ radius, vaccination was significantly more likely, whereas $12 \mathrm{~km}$ saw a very sharp decline in vaccination. ${ }^{35}$ Vaccinators coming to peoples' houses is also a viable option, one which many advocate and, in the case of Polio, mobile vaccinators played a big role. Even within fixed centres in which the EPI is being rolled out, immunization services are often open only once or twice a week. Distance to vaccination centres is also exacerbated by the low availability of opening hours. Even if immunization services were available on a daily basis, this may help to increase compliance with routine vaccinations. ${ }^{30}$

\section{Lack of Integration of the EPI and Health Worker Attitudes}

Strikingly, a very small percentage of vaccinations are covered by the private sector - around 3\%. This is important as over $80 \%$ of healthcare costs in Pakistan overall come from the private sector-mostly via out-of-pocket payments. ${ }^{36}$ The Non-Governmental Organisation (NGO) type sourcing of vaccinations may be seen as advantageous as people do not have to pay for vaccinations. This is significant within the context of high infectious disease rates amongst children in poorer areas of Pakistan. ${ }^{37}$ However, research has found that volunteer health workers have come up with problems when providing vaccines as the NGO sector is not received with enthusiasm and trust in Pakistan. Overall, private practitioners and private health infrastructure have hindered vaccines from being administered. An investigation into the reasons why there is low TT vaccination in a district of Lahore, Punjab highlighted some key issues related to existing infrastructure and its inadequacy to provide sufficient support for vaccination programmes. ${ }^{38}$ Maternal and Neonatal tetanus (MNT) elimination is implemented as part of routine vaccination all over Pakistan. Despite this, Pakistan not only ranks low in terms of vaccination but is actually labelled as class $\mathrm{C}$ by international standards which means that three to four years are needed in order to phase in elimination strategies for MNT. ${ }^{39}$ Hasnain claims that "limited health infrastructure" plays a significant role in this and low immunization coverage in general. ${ }^{38}$

The roll-out of the immunization programmes in Pakistan must be integrated into the existing health infrastructure. Routine and Polio immunizations have been set up via public-private partnerships, ${ }^{22}$ meaning that the integration of NGOs and public healthcare into this public health initiative are necessary. The overwhelming majority of health services are financed via the private sector. As a result, setting up immunization programmes that require different methods of access for the public have proved difficult. For example, it is of the opinion of many experts that the Department of Health in Pakistan did not actively 
take part in "pre-campaign activities" such as meetings, training of health workers, involvement of crucial staff such as Lady Health Workers and citing "a general lack of interest from DHMT (District Health Management Team)". ${ }^{22}$ This attitude of disinterest and unaccountability via the public health sector of Pakistan is indicative of the general structure of Pakistan's healthcare system, in which public health-funded activities have never been given priority and the financial incentive is a driving force within the health industry. ${ }^{40}$ The consequences of this mean that the integration of an essentially public health-based programme has been less than satisfactory.

Private sector involvement is therefore fast becoming a priority for vaccination programmes. It was found that the attitude of doctors in health facilities was not optimal. They would not refer children for vaccination to EPI centres and would be reluctant to integrate EPI activity into their own centres. However, the involvement of local paediatricians is vital for the chain of vaccination to go uninterrupted. Even though immunization is intended to be integrated into the public health system the role of the private sector must be included. Furthermore, if private doctors supply vaccination, this goes unrecorded from vaccination data. For this reason, recommendations have been suggested to try and integrate private clinics under government regulations for the sake of coherent vaccination implementation and monitoring. ${ }^{1}$

Following on from the conflict between the private and public sector in Pakistan, the attitude of vaccinators in Pakistan show signs of disillusionment. Mass immunization initiatives have been put in place, which includes training and implementing health workers, providing vaccines and building centres. This so far has helped achieve a mediocre level of success. Although vaccination rates have increased and the reason for this can be directly linked back to the provision of these facilities, more is needed in order to achieve global standards of public health. Dietz reports that the model of "raising coverage by the delivery of vaccines" alone is not applicable for success in low- and middle-income countries. Promotion of social factors, better management and increased awareness all need to be integrated into current healthcare infrastructure in order for vaccination programmes to achieve goals of disease elimination. ${ }^{41}$ The EPI in Pakistan also suffers from a severe shortage of human resources at the operational and also management level. The staff in provinces are both under-skilled and the ratio of EPI vaccinators to public is too low - with some areas having as few as 1 or 2 EPI staff members. This leads to the irregularity of service provision. ${ }^{15}$ Health worker resourcing is a bigger problem than vaccine provision. The argument unfolds that health workers provide not only the vaccine but also the need to create trust and relationships and therefore their attitude is a very important influencer of vaccine uptake.

Lady Health Workers (LHWs) are an integral part of the success of vaccination coverage within Pakistan. So much so that SAGE dedicate many data and research into their role. SAGE describe these LHWs as "a team, of over 100, 000 trained female community workers delivering basic health services door-to-door who hold the key to reviving Pakistan's immunization programme". ${ }^{15}$ This is a bold claim, and therefore it is important to analyze their effect on immunization uptake within communities in Pakistan, to assess whether they do lead to more successful vaccine coverage and whether they can be utilised better in the future to help Pakistan achieve globally adequate success rates.

LHWs are government employees as of 2013. Their training includes a six-month course. This training, however, is viewed by the Pakistani government as secondary to their important position within the community as trusted health workers, who can enter homes and converse openly and equally with families. The general job of an LHW includes administering vaccines, and providing general information to women about child health, hygiene, pre and post-natal care and family planning. It is estimated that each LHW serves around 1000 people. ${ }^{1}$ CSOs have complemented the role of LHWs also. They help aid in the social mobilisation of the community and have been successful at setting up vaccination camps and vaccination points, which have reduced vaccine refusals.

It may be the case however that the influence of LHWs is exaggerated and a review into the literature has shown some problems and areas that have been overlooked. Although in theory and through government reports, LHWs seem like a gold standard and revolutionary idea for immunization coverage's success, the reality may contradict this. One paper assessing maternal and neonatal tetanus in Lahore found that $90 \%$ of women did not know about the TT vaccine, claiming vaccinators had never approached their homes nor given any information about the vaccine. ${ }^{38}$ Source of information is a major contributing factor toward vaccination of women of reproductive age and for married females, the main source of information is Lady Health Workers. Therefore, it is no coincidence that in a study conducted within Peshawar it found that the two most common reasons for getting vaccinated were LHW visits to the house 
and having in-depth information about the disease. Afridi suggests that for MNT vaccination efforts, LHW communication with women of childbearing age is necessary and especially necessary at the implementation stage. ${ }^{33}$ Overwhelmingly, the literature suggests LHWs are effective and necessary for vaccination targets to be met in the future and indeed their under-usage has led to failures to reach such targets.

\section{Tried and Failed Initiatives}

There have been some initiatives that have aimed to tackle the failings of the EPI, but their level of success has been varied. Vaccination cards are good proxy within Pakistan that have developed in order to provide information and history of vaccination to families. Having a vaccine card does not assume vaccination but can give information on the attitudes and practices toward immunization within households. In one rural community in Karachi, Sindh found that only $55 \%$ of mothers had vaccination cards whilst another $23 \%$ did not even know about them. ${ }^{42}$ It has also been reported that a redesign of vaccination cards has helped increase vaccination coverage by as much as $31 \%$ in Pakistan. ${ }^{29}$ Schemes in other countries have shown that having an effective means of reporting for themselves of vaccination helped a great deal in adherence to vaccination programmes - such as the "Child Welfare Card" in Nairobi and Immunization Cards in Nigeria. ${ }^{43,44}$ The efficacy of vaccination cards has been called into question by some research, however.

Some claim it is an inefficient system of vaccination status, as they can go missing, can be inaccurate, and the system is not even computerised. Maternal recall in some cases can be a better indicator that vaccination cards; however, this is a disputed claim as recall has many problems, especially when considering many mothers do not even know the names of the diseases their children are being vaccinated against. ${ }^{30}$ In another study on TT vaccination, only $1 \%$ of mothers could produce a vaccination card. ${ }^{38}$ Assessing why this protocol has not been successful highlights important issues surrounding how to get people to adhere to routine vaccination. This monitoring system is not effective enough at creating an impact on overall vaccine coverage, as it cannot be distributed well, and people do not adhere to it correctly. Methods such as these have hindered the progression of immunization efforts and have meant that people do not adhere to routine immunization schedules properly leading to a reduced uptake of vaccine.
In the past, efforts have been made to raise immunization amongst the population; however, studies have shown these have done more harm than good. "National Immunization Days" (NIDs) were implemented all over the country to try and increase interest and demand for routine vaccination. However, it is argued that this has had a detrimental effect on routine immunization. It puts too much pressure and resources into a short period of time, polio vaccination increases but at the cost of other routine vaccinations going down in the lead-up, during and after the NID. ${ }^{1}$ Solutions like the National Immunization Days and vaccination cards show a misunderstanding of the failures of the EPI to reach adequate coverage targets. These solutions are short term in nature, trying to increase monitoring and uptake, and do not tackle the root causes of why parents do not bring their children for vaccination. Monitoring is an important part of immunization programmes, but it does not address issues such as misinformation of vaccines. Similarly, National Immunization Days, increase vaccine uptake for only one day and make an impact on polio uptake mostly. This initiative cannot address the issues of failure to take children for the second and third dose vaccinations.

\section{Female Education and Involvement}

Parents lack of knowledge on the benefits of vaccination and especially the benefits of repeat vaccination, which is also a major concern for the state of immunization within Pakistan. Reports on the leading causes of under vaccination in populations in Pakistan focus heavily on the educational level of parents. The leading cause for not taking the vaccination for Tetanus toxoid in one study within Karachi was that there was "no reported benefit" according to families $-37.5 \%$ of families reported this reason. ${ }^{25} \mathrm{In}$ addition, studies on education and its link to children's vaccination rates have repeatedly shown that within immunization campaigns it is necessary to improve parent's knowledge and awareness of vaccination practices, ideally implementing parallel educational programmes along with vaccination initiatives. ${ }^{45}$ Education on what vaccines are, what vaccine-preventable diseases their children could suffer from, and why they are important to prevent mortality may seem like obvious knowledge. However, in many cases, parents do not have access to this information. As a result, the sentiment remains that vaccination is not important and therefore children are not brought to vaccination centres, resulting in low vaccination coverage. Initiatives that advertise the purpose of the EPI are gaining 
momentum as a result of these findings. Research found that mass media campaigns were a leading "provocative factor" for vaccination compliance. ${ }^{46}$ The consequences of these media campaigns are hoped to bring knowledge and information into households that would otherwise not have access to it, making vaccination a part of the everyday conversation.

Information on VPDs is important for families in order to convince parents of the necessity of vaccination. However, further to this, female education may be a more important factor. A cross-sectional study regarding vaccine coverage in peri-urban areas in Pakistan highlighted the need for female education. The study itself centred within Karachi, which is located in the Sindh Province of Pakistan, evaluated why the tetanus vaccine was underused, via a questionnaire. The findings showed that the females in the household often made the decision as to whether children were taken for vaccination as they tend to be the primary care-giver and therefore are more influential in their children's healthcare decisions. ${ }^{25}$ In many research papers in which reasons for under vaccination are listed, a lack of mother's awareness and lack of time on the part of the mother are key reasons for under immunization. ${ }^{18,24,25}$ Within households, it was reported widely that the decision to get vaccinated was heavily weighted with the mother. Many mothers would not travel the distance to the vaccination centre, and a smaller proportion did not even know of the EPI schedule for vaccinations. The findings on the importance of the female head of the household on the decision to vaccinate are important. Lack of targeting towards mothers on the education of vaccination has meant that a key demographic that is integral to vaccination coverage has gone unnoticed. As the decision makers on this issue, their level of knowledge on how their children can benefit from vaccination could help improve rates of routine immunization.

There have been various studies on the association between maternal factors and vaccination uptake which can be applied to the framework in Pakistan. Knowledge, practice and attitudes of mothers have been studied in a broad range of countries and have mostly shown that it ispositively correlated with vaccination uptake. To provide some example of the level of knowledge, or lack thereof, within communities on Pakistan, the age to which children should be vaccinated by was asked to mothers: " $56 \%$ said 9 months, $22 \%$ said 2 years and $22 \%$ reported 3 months" and only $54 \%$ of women in the same group could name the number of diseases the EPI expected of complete vaccination. ${ }^{42} 15.8 \%$ of women reported they did not even know about vaccine-preventable diseases. One study conducted in a province in China applied a questionnaire in order to assess knowledge, attitudes and practices (KAP) of the mother toward childhood vaccination. The study found that the low level of KAP was “influenced by educational background, country's economic level, mother's age, household size and ethnic groups". ${ }^{47}$ The study also suggested that "interpersonal communication" was the main channel of knowledge and therefore should be implemented when strategizing educational programmes in the context of vaccination. This research although conducted in China can be extrapolated to relate to findings in Pakistan. One such study on the effects and causes of KAP in Pakistan was conducted in rural communities in Pakistan. The findings showed that a mother's KAP affected the health of the child as they were the primary caregiver or "first care provider". ${ }^{48}$ In many instances of child health, this was an issue, but in the context of vaccination, the link was particularly prominent and related to other areas important in child health such as stunting. Overall, the literature points to female education and awareness on this public health as imperative for the performance of the EPI to be improved and to achieve global targets of vaccine coverage.

\section{Educational Interventions}

Lack of education on vaccinations is a big issue as the decision to vaccinate lies with families, with the mother having the leading role in this. In the same questionnaire, one third of mothers did not take their children for vaccination and refused to vaccinate their children. The reasons for this included that they thought vaccination was unnecessary, that vaccination actually makes their child sick and a small proportion deemed their children too sick to receive vaccination. ${ }^{25}$ This questionnaire was conducted in one semi-urban town in Sindh, so there may be differences in attitude in other areas of Pakistan, making it difficult to extrapolate these findings to build a nationwide picture. However, it does show that these attitudes exist amongst Pakistani women and mothers to some extent. This should be an area of focus for future immunization programmes to target. Therefore, the challenge for immunization service providers is to offer parents balanced and comprehensive information about both the risks and benefits of immunization during counselling sessions.

Educational interventions will be necessary in the future in order to combat low vaccine uptake by families. 
One such intervention was simple but proved statistically effective. Three "pictorial messages" were given to families by community-based health workers already in place within the healthcare system. When compared with a group that was given only the routine public health advice on vaccines by LHWs, it was found that rates of immunization increased in the intervention group. DPT4 and Hepatitis B vaccine completion rose by $39 \%$ overall, when other variables were taken into account. ${ }^{13}$ The study was conducted within a community that had low literacy and high child mortality, and therefore the outcomes show that simple education-based intervention can work in this repeatable model as the context is similar in many communities in Pakistan. Interventions that address the need of low literacy populations are necessary as vaccination and infectious diseases are a health care phenomenon that cannot wait for overhauls of social and educational change. ${ }^{49}$ LHWs are a system of community health workers already in place that can be utilised in order to implement these educational programmes. Overall, the literature on the correlation between female education and awareness and vaccination uptake for children shows that this should be the main area of focus for the future of immunization in Pakistan.

\section{Parental Trust}

Similar to education, the information or misinformation given to parents shapes vaccination coverage and has played a big role in the under vaccination of the population. For example, research shows that the demand for OPV at the moment is high, and that there is high parental support for it. However, it is possible that this demand may fall in the future as misconceptions about vaccination and VPDs become more common and are not addressed by immunization programmes. ${ }^{50}$ Trust amongst parents plays an important role for vaccination efforts. Within low conflict areas of Pakistan which include Sindh, KP and Punjab, it was found that $61 \%$ of parents trust vaccinators. However, in high conflict areas which includes FATA, this number was much lower at $28 \%$. This lack of trust extends to further attitudes which put vaccination in jeopardy, such as the view or rumour that vaccination causes sterilisation amongst boys and girls.

As well as the lack of parental education, making mothers unaware of vaccinations and their benefits, there is a growing phenomenon that parents believe vaccination is unsafe to varying degrees. ${ }^{13}$ Within the developed world this is a phenomenon that gripped public health in the early twentieth century, following the infamous measles, mumps and rubella (MMR) scandal and subsequent anti-vaccination movements. Jefferson concludes that distrust of science and greater attention to risk have helped fuel this concept amongst parents. He suggests three "priorities" for the future to combat concerns around vaccination which include; universal definitions for vaccine adverse effects, better epidemiological information and rapid communication of current research on causal links. ${ }^{51}$ This model is very easily applied within the context of high-income countries and most of Jefferson's data were conducted using European models of public health. Within low-income countries such as Pakistan however, although a similar phenomenon exists, the reasons why may need to be evaluated differently. However, research has shown that the adverse effects of vaccination in Pakistan are reported more frequently than the positive outcomes, which helps to contribute to parent's unwillingness to take their children for vaccination. ${ }^{13}$ Nisar reported in 2010 that $11.5 \%$ of mothers thought that vaccines would harm their children. ${ }^{42}$ This is an example of how misinformation about vaccination has had detrimental effects on vaccine coverage. Education on the importance of vaccination and re-education to overcome this misinformation is vital in order to make parents bring their children to EPI centres and increase vaccine coverage along with the GVAP targets.

\section{Aid Clustering and the Polio Problem}

The burden of vaccine-preventable diseases within Pakistan over the past decade has been overshadowed by polio. Pakistan is one of the three remaining countries in which Polio is endemic. ${ }^{52}$ Indeed, the eradication of polio in Pakistan has seen unique setbacks when compared with other VPDs and it is for this reason that it is beneficial to assess polio somewhat separately. Some reports put polio vaccination coverage as low as $50 \%$ in some areas. ${ }^{53}$ In the eyes of the international community, Pakistan's failure to eliminate polio contributes greatly to its image of failure with regard to communicable diseases and public health. If elimination is achieved the public health status of Pakistan could be greatly improved, and lessons learned from this achievement can help other vaccination efforts achieve success. The WHO reports that Supplementary Immunization Activities (SIAs) have successfully resulted in the elimination of polio globally which is the leading reason for high polio coverage, and cases of poliomyelitis have decreased by $99 \%$ since $1988 .{ }^{54}$ The failure of polio eradication in Pakistan, however, is creating problems for the "global 
scenario for a polio free world". 55 The extent of this VPD in the global forum has led to multiple travel bans placed for Pakistan by the WHO and other countries. It is clear therefore that the polio status of Pakistan is an international concern, one that overshadows the health profile of the country greatly.

The majority of polio cases (69\%) come from the FATA, making it the major poliovirus reservoir of Pakistan. ${ }^{56}$ However, the international focus may be skewed towards this region as there have been confirmed cases of polio in low violence zones in Punjab, Sindh and Khyber Pakhtunkhwa. ${ }^{55}$ Polio vaccination efforts can reveal why vaccination efforts in Pakistan uniquely have failed to work, despite implementing similar strategies as other countries. It was found that $10-20 \%$ of children who receive the initial first dose of trivalent polio vaccine, do not receive their second and third doses. This is due to the inefficiencies of the EPI, where service delivery and provision of information has led to these dropout rates. ${ }^{53}$ This has implications further down as these dropout rates decrease the proportion of fully immunized individuals, making the impact of vaccination on polio transmission useless. ${ }^{57} \mathrm{We}$ can, therefore, assess the extent to which polio under vaccination is prevalent in Pakistan. With regard to the global standards of public, elimination is the aim. Breakouts put the international community at risk also and therefore it is a healthcare priority for Pakistan.

Immunization within Pakistan has been split into two camps because of the narrow focus on polio eradication. There are two parallel initiatives going on in Pakistan one for routine immunization and one for polio immunization. As a result, these two often become competing activities at the local context. Thus, "polio fatigue" has ensued, in which immunization workers feel that resources and focus are not adequately given to routine immunization as a result. The separation of these two vaccination efforts can be seen in the separate "Global Polio Eradication Initiative" (GPEI) which was launched in 1988. The initiative worldwide reduced cases of Polio by $99 \% .{ }^{52}$ However, Pakistan is one of the last countries in which it is endemic. In 2014, 359 cases were reported globally - of which 306 cases were found in Pakistan.

The focus on polio has drawn attention away from other VPDs and may contribute to the low vaccination coverage of routine vaccination. On the other hand, although polio is a necessary barrier to overcome for the EPI it overshadows other diseases which account for more deaths annually than polio. Owais et al claim that
"Although the number of polio cases decreased to 58 in 2012 through better organized supplementary immunization campaigns, country-wide measles outbreaks with over 15,000 cases and several hundred deaths in 2012-13 underscore sub-optimal EPI performance in delivering routine immunizations." The burden of measles on child health has not had the same depth of research and focus than polio yet it is a bigger contributor to mortality. ${ }^{10}$ It cannot be underestimated that polio is an important disease burden in Pakistan. However, polio campaigns may be taking away resources and research from routine immunization making it more difficult to achieve GVAP goals.

\section{Cultural Resistance}

Perhaps, one of the main factors that separates Pakistan and has been a unique reason as to why immunization programmes have failed to reach international targets is because of the role and influence of Islamic clerics in communities who make judgements on healthcare. The leading secondary reason for inadequate vaccine uptake is religious taboos. ${ }^{46}$ Religious taboo is a somewhat un-useful term for the purpose of analysis as the influence of religion on everyday life within Pakistan is complicated and not well researched. The religious influence on the uptake of vaccination is part of the bigger "cultural resistance". ${ }^{22}$ This religious aspect is fuelled by religious leaders who have places within the community hierarchy as opinion-makers who can sustain local myths, such as vaccine side effects and vaccine-induced infertility. ${ }^{22}$ These so-called myths and rumours stem from a mistrust of foreign providers and tensions between Pakistan and the West. Generally, religious clerics in communities have, in the past, stopped people taking up the polio vaccine by calling it a Western ploy to sterilize Muslim children and put an end to Islamic progeny. ${ }^{58}$ This is a strong phenomenon that has developed and has contributed to the refusal to take the vaccine, leading to low coverage. Hence, in order to alleviate the problem of poor community uptake of vaccinations, religious leaders must be engaged in the EPI programmes, so they can support the public and vaccine providers in the most personal context within the community.

The influence of religion on health decisions cannot be easily reversed or changed, and it is, therefore, necessary for the government and NGOs to co-operate with these religious and social structures in the different forms they take. $^{46}$ The Centres for Disease and Control Prevention (CDC) has acknowledged how the unique influence of religion has impacted vaccination status in Pakistan. The 
high conflict areas in Pakistan have seen rises in Wild Type Polio (WPV) transmission as a result. The CDC suggests implementing "national emergency action plans" in order to enhance safety for vaccination workers and those who want to take the vaccine in conflict-affected areas. ${ }^{59}$ The traditional strategies of immunization campaigns which emphasise foreign aid lead to the fallout of vaccination uptake. Hajaj suggests that international agencies should rather emphasise "inclusive ownership" in order to shift the antiWestern sentiment and re-educate religious community leaders on the public benefit to vaccination. ${ }^{60}$ This is a very important part of Pakistan's path to achieve acceptable rates of vaccine coverage, once we see how much these community leaders affect vaccine uptake. Alexander, in his assessment of the dangers associated with vaccination status in Pakistan, reports that 350000 children in FATA have not received polio vaccine since mid-2012, because local authorities have banned polio vaccination. In other areas of Pakistan, supplementary immunization activities (SIAs) have also been hindered by attacks on polio workers. ${ }^{61}$ From this, it can be concluded that the influence of Islamic leaders can be deleterious for the population of Pakistan and the progressions of immunization programs.

The three leading causes of under vaccination amongst eligible children for polio were "(1) extremist-related security issues; (2) parental refusal of vaccination; and (3) concerns about the efficacy and safety of the polio vaccine". ${ }^{6}$ We can see here the overlap with other vaccine-preventable diseases with parental refusal and safety concerns however the involvement of militancy in the polio issue is unique. The security issue for vaccination health workers should not be taken lightly, as it has led to nearly 200000 eligible children going unvaccinated in tribal areas of Waziristan during the 2011-2010 anti-polio campaigns alone. ${ }^{57}$ Religious influence takes a further serious turn when the Pakistan Taliban's influence is considered. Their effect on the number of children vaccinated is shocking. They have implemented the blocking of polio immunization campaigns in FATA (one of the main polio reservoirs in Pakistan) which meant a further 200000 children were unable to be vaccinated. ${ }^{56}$ From this, we can see a direct cause of under vaccination, due to the feeling of mistrust toward vaccinations and programs. This became an important talking point in the effort to reach vaccination targets.

\section{Conclusion}

Pakistan retains some fundamental issues that halt the progression of vaccination services. Firstly, some of these problems arise from limitations due to the healthcare infrastructure. Generally speaking, district and province divisions arise from facets of history and culture and is something that is difficult to change. For the EPI to work to its full potential, it must integrate into the existing health infrastructure well. This means a common incentive, monitoring and cohesion is needed between private practitioners, health workers from NGOs and health workers from other streams of healthcare such as LHWs.

On balance, the EPI and polio campaigns have not completely "failed". However, the rate at which Pakistan is achieving targets is slow and therefore places the country in a disadvantaged view from the perspective of global health actors. Furthermore, the prevalence of polio in Pakistan has shone a negative light on the country and brought much focus on the issue of immunization. The argument remains that the inability of immunization programmes to be successfully rolled out nationwide stem largely from problems within Pakistan that make it difficult to implement such initiatives. However, it is the responsibility of both global health actors and the government of Pakistan to overcome this by addressing the discussed obstacles hindering the implementation of immunization efforts.

\section{Author Contributions}

All authors contributed to data analysis, drafting and revising the article, gave final approval of the version to be published, and agree to be accountable for all aspects of the work.

\section{Disclosure}

The authors report no conflicts of interest in this work.

\section{References}

1. Mangrio NK, Alam MM, Shaikh BT. Is expanded programme on immunization doing enough? Viewpoint of health workers and managers in Sindh, Pakistan. J Pak Med Assoc. 2008;58(2):64-67.

2. Anand S, Barnighausen T. Health workers and vaccination coverage in developing countries: an econometric analysis. Lancet. 2007;369 (9569):1277-1285. doi:10.1016/S0140-6736(07)60599-6

3. Unicef. GIVS: Global Immunization Vision and Strategy, 2006-2015. Givs; 2005.

4. Mansuri G, Rao V. Community-based and -driven development: a critical review. World Bank Res Obs. 2004;19:1-39. doi:10.1093/ wbro/lkh012

5. The World Bank Group. World Bank Annual Report 2017; 2017. doi:10.1596/978-1-4648-1119-7

6. Mahmud G, Zaman F, Jafarey S, Khan RL, Sohail R, Fatima S. Achieving millennium development goals 4 and 5 in Pakistan. BJOG. 2011;118:69-77. doi:10.1111/j.1471-0528.2011.03114.x 
7. Mehnaz A. Infectious diseases in children-still leads. J Pak Med Assoc. 2009;59(7):425-426.

8. Bhutta ZA, Hafeez A, Rizvi A, Ali N, Khan A, Ahmad F. Reproductive, maternal, newborn, and child health in Pakistan: challenges and opportunities. Lancet. 2013;381:2207-2218. doi:10.1016/ S0140-6736(12)61999-0

9. Hussain H, Waters H, Omer SB, et al. The cost of treatment for child pneumonias and meningitis in the Northern Areas of Pakistan. Int J Health Plann Manage. 2006;21(3):229-238. doi:10.1002/hpm. 847

10. Owais A, Khowaja AR, Ali SA, Zaidi AKM. Pakistan's expanded programme on immunization: an overview in the context of polio eradication and strategies for improving coverage. Vaccine. 2013;31 (33):3313-3319. doi:10.1016/j.vaccine.2013.05.015

11. Lim SS, Stein DB, Charrow A, Murray CJL. Tracking progress towards universal childhood immunisation and the impact of global initiatives: a systematic analysis of three-dose diphtheria, tetanus, and pertussis immunisation coverage. Lancet. 2008;372(9655):20 31-2046. doi:10.1016/S0140-6736(08)61869-3

12. Loevinsohn B, Hong R, Gauri V. Will more inputs improve the delivery of health services?: analysis of district vaccination coverage in Pakistan. Int $J$ Health Plann Manage. 2006;21(1):45-54. doi: $10.1002 / \mathrm{hpm} .824$

13. Owais A, Hanif B, Siddiqui AR, Agha A, Zaidi AKM. Does improving maternal knowledge of vaccines impact infant immunization rates? A community-based randomized-controlled trial in Karachi, Pakistan. BMC Public Health. 2011;11(1):239. doi:10.1186/14712458-11-239

14. Keja K, Chan C, Hayden G, Henderson RH. Expanded programme on immunization. World Health Stat Q. 1988;41(2):59-63.

15. WHO. Global Vaccine Action Plan 2011-2020; 2017.

16. Ali SZ. Health for all in Pakistan: achievements, strategies and challenges. East Mediterr Health J. 2000;6(4):832-837.

17. Closser S. "We can't give up now": global health optimism and polio eradication in Pakistan. Med Anthropol. 2012;31(5):385-403. doi:10.1080/01459740.2011.645927

18. Naz D, Zafar Siddiqui N, Bano A, et al. A cross sectional survey: attitude towards adult vaccination in Karachi-Pakistan. Int $J$ Adv Res. 2015;3:03.

19. Buse K, Walt G. Global public-private partnerships: part I - a new development in health? Bull World Health Organ. 2000;78(4):54 9-561.

20. Siddiqui R, Afridi U, Haq R. Determinants of expenditure on health in Pakistan. Pak Dev Rev. 1995;34(4III):959-970. doi:10.30541/ v34i4iiipp.959-970

21. Lambo JA, Nagulesapillai T. Neonatal tetanus elimination in Pakistan: progress and challenges. Int J Infect Dis. 2012;16(12): e833-e842. doi:10.1016/j.ijid.2012.07.015

22. Husain S, Omer SB. Routine immunization services in Pakistan: seeing beyond the numbers. East Mediterr Heal J. 2016;22(3):201. doi: $10.26719 / 2016.22 .3 .201$

23. Kols A, Gorar Z, Sharjeel M, et al. Provincial differences in levels, trends, and determinants of child-hood immunization in Pakistan EMHJ. 2018;24(4):333-344. doi:10.26719/2018.24.4.333

24. Hasan Q, Bosan AH, Mohamud K. A review of EPI progress in pakistan towards achieving coverage targets: present situation and the way forward. EMHJ. 2010; Vol(16 Suppl):S31-S38. doi:10.26 719/2010.16.Supp.31

25. Siddiqui FJ, Rabbani F, Hasan R, Nizami SQ, Bhutta ZA. Typhoid fever in children: some epidemiological considerations from Karachi, Pakistan. Int J Infect Dis. 2006;10(3):215-222. doi:10.1016/j.ijid.20 05.03.010

26. Rehman SU, Siddiqui AR, Ahmed J, et al. Coverage and predictors of routine immunization among 12-23 months old children in disaster affected communities in Pakistan. Int J Health Sci (Qassim). 2017;11(1):1-6.
27. Unicef. The state of the world's children, 2007: women and children: the double dividend of gender equality. Choice Rev Online. 2007;45 (02):45-1173. doi: $10.5860 /$ choice. $45-1173$

28. Zaidi AKM, Khan H, Lasi R, Mahesar W. Surveillance of pneumococcal meningitis among children in Sindh, Southern Pakistan. Clin Infect Dis. 2009;48(Supplement 2):S129-S135. doi:10.1086/596491

29. Usman HR, Akhtar S, Habib F, Jehan I. Redesigned immunization card and center-based education to reduce childhood immunization dropouts in urban Pakistan: a randomized controlled trial. Vaccine. 2009;27(3):467-472. doi:10.1016/j.vaccine.2008.10.048

30. Rasheed M, Akram U, Asif N, Ahmed K, Zafar S, Mumtaz S. Expanded Programme of Immunization (EPI) status among children of factory workers. JIMDC. 2014;(3):62-66. https://jimdc.org.pk/ jimdc/Volumes/3-2/Expanded Programme of Immunization.pdf.

31. Chandir S, Siddiqi D, Dharma V, et al. Zindagi Mehfooz (Safe Life) Digital Immunization Registry: Leveraging Low-Cost Technology to Improve Immunization Coverage and Timeliness in Pakistan (Preprint). 2018. doi:10.2196/preprints. 11770

32. Kazi AM. The role of mobile phone-based interventions to improve routine childhood immunisation coverage. Lancet Glob Health. 2017;5(4):e377-e378. doi:10.1016/S2214-109X(17)30088-8

33. Afridi NK, Hatcher J, Mahmud S, Nanan D. Coverage and factors associated with tetanus toxoid vaccination status among females of reproductive age in Peshawar. J Coll Physicians Surg Pak. 2005;15 (7):391-395. doi:07.2005/JCPSP.391395

34. Khowaja AR, Zaman U, Feroze A, Rizvi A, Zaidi AKM. Routine EPI coverage: subdistrict inequalities and reasons for immunization failure in a rural setting in Pakistan. Asia Pac J Public Health. 2015;27 (2):NP1050-NP1059. doi:10.1177/1010539511430850

35. Naeem M, Adil M, Abbas SH, et al. Coverage and causes of missed oral polio vaccine in urban and rural areas of Peshawar. J Ayub Med Coll Abbottabad. 2011;23(4):98-102.

36. Shaikh BT. Private sector in health care delivery: a reality and a challenge in Pakistan. J Ayub Med Coll Abbottabad. 2015;27 (2):496-498.

37. Hyder AA, Morrow RH. Applying burden of disease methods in developing countries: a case study from Pakistan. Am J Public Health. 2000;90(8):1235.

38. Hasnain S, Sheikh NH. Causes of low tetanus toxoid vaccination coverage in pregnant women in Lahore district, Pakistan. East Mediterr Heal J. 2007;13:1142-1152. doi:10.26719/2007.13.5.1142

39. Owusu-Darko S, Diouf K, Nour NM. Elimination of maternal and neonatal tetanus: a 21 st-century challenge. Rev Obstet Gynecol. 2012;5(3-4):e151-e157.

40. Haq Z, Iqbal Z, Rahman A. Job stress among community health workers: a multi-method study from Pakistan. Int $J$ Ment Health Syst. 2008;2(1):15. doi:10.1186/1752-4458-2-15

41. Dietz V, Cutts F. The use of mass campaigns in the expanded program on immunization: a review of reported advantages and disadvantages. Int J Heal Serv. 1997;27(4):767-790. doi:10.2190/ QPCQ-FBF8-6ABX-2TB5

42. Nisar N, Mirza M, Hafeez Qadri M. Knowledge, attitude and practices of mothers regarding immunization of one year old child at Mawatch Goth, Kemari Town, Karachi. Pak J Med Sci. 2010;26:183-186.

43. Babalola S, Lawan U. Factors predicting BCG immunization status in northern Nigeria: a behavioral-ecological perspective. $J$ Child Health Care. 2009;13(1):46-62. doi:10.1177/1367493508098380

44. Owino LO, Irimu G, Olenja J, Meme JS. Factors influencing immunisation coverage in Mathare Valley, Nairobi. East Afr Med J. 2009;86(7):323-329.

45. Al-lela OQB, Bahari MB, Salih MRM, Al-Abbassi MG, Elkalmi RM, Jamshed SQ. Factors underlying inadequate parents' awareness regarding pediatrics immunization: findings of cross-sectional study in Mosul- Iraq. BMC Pediatr. 2014;14:29. doi:10.1186/1471-243114-29 
46. Sheikh A, Iqbal B, Ehtamam A, et al. Reasons for non-vaccination in pediatric patients visiting tertiary care centers in a polio-prone country. Arch Public Health. 2013;71(1):19. doi:10.1186/07787367-71-19

47. Wang Y, Wang Y, Zhang J, Kang C, Duan P. Status of mother's KAP on child immunization in minority areas, Guizhou Province. Beijing Da Xue Xиe Bao. 2007;39(2):136-139.

48. Agha A, White F, Younus M, Kadir MM, Alir S, Fatmi Z. Eight key household practices of integrated management of childhood illnesses (IMCI) amongst mothers of children aged 6 to 59 months in Gambat, Sindh, Pakistan. J Pak Med Assoc. 2007;57(6):288-293.

49. Mitchell S, Andersson N, Ansari NM, Omer K, Soberanis JL, Cockcroft A. Equity and vaccine uptake: a cross-sectional study of measles vaccination in Lasbela District, Pakistan. BMC Int Health Hum Rights. 2009;9(S1):S7. doi:10.1186/1472-698X-9-S1-S7

50. Taylor SAJ. Culture and behaviour in mass health interventions: lessons from the global polio eradication initiative. Crit Public Health. 2015;25(2):192-204. doi:10.1080/09581596.2014.895799

51. Jefferson TOM, Real or perceived adverse effects of vaccines and the media - a tale of our times. J Epidemiol Community Health. 2000;54 (6):402 LP- 403. doi:10.1136/jech.54.6.402

52. Khan MT, Zaheer S, Shafique K. Maternal education, empowerment, economic status and child polio vaccination uptake in Pakistan: a population based cross sectional study. BMJ Open. 2017;7(3): e013853. doi:10.1136/bmjopen-2016-013853

53. Shah M, Khan MK, Shakeel S, et al. Resistance of polio to its eradication in Pakistan. Virol J. 2011;8:457. doi:10.1186/1743$422 \mathrm{X}-8-457$
54. Kazi AN. Measles epidemic exposes inadequate vaccination coverage in Pakistan. BMJ. 2013;346:f245-f245. doi:10.1136/bmj.f245

55. Kazi AM, Murtaza A, Khoja S, Zaidi AK, Ali SA. Monitoring polio supplementary immunization activities using an automated short text messaging system in Karachi, Pakistan. Bull World Health Organ. 2014;92(3):220-225. doi:10.2471/BLT.13.122564

56. Mohammadi D. The final push for polio eradication? Lancet. 2017;380(9840):460-462. doi:10.1016/S0140-6736(12)61279-3

57. Fetene N. Determinant factors for implementing polio eradication activities under security compromised settings of Pakistan. J Trop Dis Public Health. 2013;2. doi:10.4172/2329-891X.1000127

58. Salim F. Culture, politics, and religion: exploring resistance to vaccinations in South Asia. Hum Welf. 2012;1(1):91-103.

59. Centers for Disease Control and Prevention (CDC). Progress toward interruption of wild poliovirus transmission-worldwide, January 2011March 2012. MMWR Morb Mortal Wkly Rep. 2012;61(19):353-357.

60. Hajaj C, Reitano T. Casualties of war: polio and the Golden Millimeter. Glob Heal Gov. 2016;10(2).

61. Alexander JPJ, Zubair M, Khan M, Abid N, Durry E. Progress and peril: poliomyelitis eradication efforts in Pakistan, 1994-2013. J Infect Dis. 2014;210(suppl 1):S152-S161. doi:10.1093/infdis/ jiu450

62. Ahmad SO, Yousuf F, Bux AS, Abu-Zaid A. Pakistan: the final frontier for global polio eradication. $J$ Epidemiol Community Health. 2015;70(2):109-110. doi:10.1136/jech-2015-205530
Risk Management and Healthcare Policy

\section{Publish your work in this journal}

Risk Management and Healthcare Policy is an international, peerreviewed, open access journal focusing on all aspects of public health, policy, and preventative measures to promote good health and improve morbidity and mortality in the population. The journal welcomes submitted papers covering original research, basic science, clinical \& epidemiological studies, reviews and evaluations,

\section{Dovepress}

guidelines, expert opinion and commentary, case reports and extended reports. The manuscript management system is completely online and includes a very quick and fair peer-review system, which is all easy to use. Visit http://www.dovepress.com/testimonials.php to read real quotes from published authors. 